\title{
Poly-phosphate increases SMC differentiation of mesenchymal stem cells on PLGA-polyurethane nanofibrous scaffold
}

\author{
Hossein Rezaei - Zahra Rezaie - Seyed Morteza Seifati • Abdolreza Ardeshirylajimi • \\ Abbas Basiri • Mohammad Taheri - Mir Davood Omrani
}

Received: 5 January 2020/Accepted: 4 May 2020/Published online: 9 May 2020

(C) Springer Nature B.V. 2020

\begin{abstract}
The use of bioactive scaffolds in tissue engineering has a significant effect on the damaged tissue healing by an increase in speed and quality of the process. Herein, electrospinning was applied to fabricate composite nanofibrous scaffolds by Poly lactic-co-glycolic acid (PLGA) and Polyurethane (PU) with and without poly-phosphate (poly-P). Scaffolds were characterized morphologically by scanning electron microscope (SEM), and their biocompatibility was also investigated by SEM, protein adsorption,
\end{abstract}

H. Rezaei

International Campus Yazd University, Yazd, Iran

Z. Rezaie · M. Taheri $(\bowtie)$

Urogenital Stem Cell Research Center, Shahid Beheshti University of Medical Sciences, No. 23, Shahid Labbafi

Nejad Educational Hospital, Amir Ebrahimi St, Pasdaran Ave, Tehran, Iran

e-mail: mohammad.taheri@sbmu.ac.ir

\section{S. M. Seifati}

Medical Biotechnology Research Center, Ashkezar

Branch, Islamic Azad University, Ashkezar,

Yazd, Iran

\section{A. Ardeshirylajimi}

Edward A. Doisy Department of Biochemistry and Molecular Biology, Saint Louis University School of Medicine, Saint Louis, MO, USA

A. Basiri · M. D. Omrani ( $\square)$

Urology and Nephrology Research Center, Shahid Beheshti University of Medical Sciences, Tehran, Iran e-mail: davood_omrani@sbmu.ac.ir cell attachment and survival assays. The applicability of the scaffolds for bladder tissue engineering was also evaluated by culturing mesenchymal stem cells (MSCs) on the scaffolds and their differentiation into smooth muscle cell (SMC) was studied at the gene and protein levels. The results demonstrated that scaffold biocompatibility was increased significantly by loading poly-P. SMC related gene and protein expression level in MSCs cultured on poly-P-loaded scaffold was also increased significantly compared to those cells cultured on empty scaffold. It can be concluded that poly-P hasn't also increased scaffold biocompatibility, but also SMC differentiation potential of MSCs was also increased while cultured on the poly-P containing scaffold compared to the empty scaffold. Taken together, our study showed that PLGA-PU-poly-P alone and in combination with MSCs has a promising potential for support urinary bladder smooth muscle tissue engineering.

Keywords PLGA/PU/poly-P · Smooth muscle cell · Mesenchymal stem cells · Bladder tissue engineering

\section{Introduction}

Inorganic polyphosphate (poly-P) is a long linear polymer of orthophosphates $(\mathrm{Pi})$ with size up to 1000 $\mathrm{Pi}$ roots and in addition to being found in cells, this polymer chain also exists extracellularly in the blood 
plasma (Hassanian et al. 2017). Poly-P may act as a storage molecule for energetic phosphate, calcium chelator or other divalent cations, as an opposing ion for amino acids in vacuoles, as a regulator of intracellular levels of adenylate nucleotides through inhibition (Lorenz et al. 2012; Schröder and Müller 2012). Adenylate kinase, used as a phosphate donor for some glucose kinases, or may play a role in apoptosis or response of cells to osmotic pressure and $\mathrm{pH}$ pressure. Recent studies have shown also the role of poly-P in bone formation, cell proliferation, apoptosis, metastatic tumors, blood clots, and the regulation of inflammation (Ardeshirylajimi et al. 2018; Dedkova and Blatter 2014; Solesio et al. 2016). These poly-P roles are mediated by stimulation of intracellular signaling pathways. Poly-P activates receptors on cell membranes such as integrin, fibroblast growth factors (FGF), P2Y1 and also induces nitric oxide synthase expression. By inducing FGF, it activates ERK/p38 and mitogen-activated protein kinase (MAPK)-mediated signaling (Hassanian et al. 2016). It also confirmed that polyP activates the mTOR signaling pathway in cancer cells and the Wnt signaling pathway in osteogenesis. One of the most important roles of poly-P is in the transport of membrane ions, where, the channel formed by poly$\mathrm{P}$ and calcium ion and Polyhydroxybutyrate (polyP/ $\left.\mathrm{Ca} 2^{+} / \mathrm{PHB}\right)$ plays an important role in increasing membrane permeability (Dedkova and Blatter 2014).

The bladder smooth muscle can be disturbed by many factors that can change people's lifestyle and disrupt their routine life. No definitive treatment for these disorders has been reported so far, and current therapies only partially alleviate some of the patients' problems and do not completely cure them (Franco 2012; Hoag and Gani 2015; Schmidt 2008). In this because, scientists have tried to use tissue engineering (TE) for introduce alternative therapies for bladder disorders. TE is the science of designing and producing new tissues for damaged organs and tissues or repairing tissue lesions and improving organ function through the design and fabrication of 3D scaffolds that can be cultured within cells. There are three key factors in TE: signaling macromolecules, stem cell, and scaffold as an alternative extracellular matrix (ECM) (Griffith and Naughton 2002; Lanza et al. 2011). Scaffolds are used as a synthetic cellular ECM to generate growth signals and guide cell differentiation and development of new tissue. Space and mechanical stability created by scaffold through transverse connections can be very useful in repairing irregular defects and formation of new tissue (Amani et al. 2019). Polymeric nano-fibers can be prepared in a variety of ways, including phase separation, synthetic molding, self-assembly, drawing, and electrospinning (Alghoraibi and Alomari 2018). Electrospinning is one of the most widely used, because is a relatively simple way to produce nanoscale or sub-micron scale fibrous scaffolds that are morphologically similar to the natural ECM (Wang et al. 2009). Electrospun fiber matrices can have a high specific surface area due to the possibility of creating very thin diameters and thus can efficiently release biomolecules (Subbiah et al. 2005). Also, the loose bond between the fibers can be useful for tissue growth and cell migration. In addition, electrospinning is applicable to all soluble polymers, these polymers are chemically changeable and can also be added to bases of particles, including carbon and complex particles such as proteins, growth factors and enzymes (Sill and von Recum 2008). Thus, electrospun nanofibrous scaffolds, in addition to acting as a synthetic ECM underlying scaffolds, they can also serve as carriers for the controlled delivery of an active biomolecule. In this technology, the scaffold can release a specific factor for a certain period of time, which can exert its biological function upon release. This will both slowly discharge the required amount of material in place and reduce the wastage of additional costs (Khalf and Madihally 2017).

Stem cells/cells as another key factor of TE constructs can be selected from various options, but Mesenchymal stem cells (MSCs) have priority among stem cells since their advantages in tissue regeneration and immunomodulation was confirmed in human and clinical trials. MSCs participate has been confirmed in the repair of tissues of mesenchymal origin such as bone, cartilage, muscle, tendon and adipose (Ardeshirylajimi et al. 2020; Golchin et al. 2019; Mao et al. 2017).

In this study, electrospun nanofibrous scaffolds were fabricated from Poly lactic-co-glycolic acid (PLGA) and Polyurethane (PU) while incorporated with poly-P to investigate poly-P possible role in support of smooth muscle cells (SMC) differentiation potential of human MSCs. PLGA and PU were selected due to their unique characteristics such as hydrophilicity, proper biodegradability, proper 
temperature and $\mathrm{pH}$ sensitive drug delivery and release system, nontoxicity and blood compatibility (Laschke et al. 2009; Park et al. 2007; Zhu et al. 2014). After structurally and morphologically characterization of the scaffolds, their biocompatibility was also studied by protein adsorption, cell attachment and cells survival assays. Finally SMC differentiation potential of human MSCs while cultured on polyPincorporated scaffold and scaffold without polyP was studied at the gene and protein levels using real-time reverse transcription polymerase chain reaction (RTPCR) and immunocytochemistry.

\section{Materials and methods}

\section{Composite electrospun scaffold fabrication}

PU $(\mathrm{Mw}=110,000$, Cardio Tech. Intern. Japan $)$ and PLGA $(\mathrm{Mw}=45,000$, Jinan Daigang Biomaterial Co., Ltd. Shandong, China) were purchased and then PLGA/PU solution blend of 50/50 weight ratios were prepared by dissolve in a solvent that prepared by the mixture of tetrahydrofuran (THF) and $N, N$-dimethylformamide (DMF) (60/40, v/v). To poly-P incorporated PLGA/PU nanofibrous scaffold, $25 \mu \mathrm{g}$ of polyP70 was added to the PLGA/PU solution and stirred for $3 \mathrm{~h}$ before electrospinning. After that prepared solutions were fed to $5 \mathrm{~mL}$ needle blunted syringes and then electrospinning was done according to the following parameters including, voltage: $18 \mathrm{kV}$, flow rate: $0.6 \mathrm{~mL} / \mathrm{h}$ and needle was $20 \mathrm{~cm}$ away from the collector. Prior to scaffolds characterization and cell seeding, scaffolds were treated by $\mathrm{O}_{2}$-Plasma to increase surface hydrophilicity with increasing active functional residues by placed in a cylindrical quartz reactor (Diener Electronics, Germany) while its pressure was 0.4 mbar for $10 \mathrm{~min}$.

Nanofibrous scaffolds morphological study

To morphological and structural study of the fabricated scaffolds, they were fixed when cells cultured on them by glutaraldehyde $(2.5 \%)$ and then dehydrated by rinsing in ethanol serial dilution from $50^{\circ}$ to $100^{\circ}$. Fixed scaffolds after drying and intact scaffolds (without cells) were sputtered with a thin gold layer and then visualized using a scanning electron microscope (S-4500; Hitachi, Japan).
Contact angle

Hydrophilicity of the fabricated nanofibrous scaffolds was studied by water contact angle evaluation after plasma treatment using a G10 Kruss contact angle goniometer after falling a drop of water on them during $10 \mathrm{~s}$ at room temperature.

Mechanical study

To mechanical property evaluation, Galdabini testing equipment was applied to perfume tensile test for fabricated nanofibrous composite scaffolds. Scaffolds were cut into $10 \mathrm{~mm} \times 60 \mathrm{~mm} \times 0.11 \mathrm{~mm}$ pieces and put into the equipment and test was run at a speed of $50 \mathrm{~mm} / \mathrm{min}$.

\section{Protein adsorption assay}

Protein adsorption capability of the fabricated nanofibrous scaffolds was studied according to our previous reported protocol (Ghorbani et al. 2015). Scaffolds with certain dimension and weight were sterilized using UV irradiation (1 h) under laminar hood and then their protein adsorption affinity was measured by a stepwise protocol. First PBS/fetal bovine serum (FBS, $1 \% \mathrm{v} / \mathrm{v}$ ) solution-that its total protein content was quantified by a protein assay kit (BCA; Pierce, Rockford, IL, USA) previously-was added to the scaffolds and kept at $37{ }^{\circ} \mathrm{C}$ for $1 \mathrm{~h}$. After that, scaffolds were removed and put on the PBS/sodium dodecylsulfate (SDS, $2.0 \mathrm{wt} \%$ ) for $20 \mathrm{~h}$. After removing scaffolds from this solution, its total protein content was also measured and reported in $\mu \mathrm{g}$ proteins per $\mathrm{mm}^{3}$ of scaffold while compared with optical densities (OD) obtained from a BSA calibration in SDS/PBS at $530 \mathrm{~nm}$ using a spectrophotometer.

\section{Mesenchymal stem cells isolation and characterization}

To adipose derived MSCs preparation, first, abdominal fat tissue was obtained from patients underlying liposuction surgery (Taleghani Hospital, Tehran, Iran) based on the medical ethics committee guidelines of Iranian Ministry of Health and transferred to the laboratory under aseptic and sterile condition by put in DMEM medium content antibiotics and antifungals. After that MSCs were isolated enzymatically from fat 
tissues and then characterized by differentiating to the adipocyte-like and osteoblast-like cells according to our previous studies (Ardeshirylajimi et al. 2016; Ghiaee et al. 2017). Flow cytometry analysis was also performed to more characterization of the isolated MSCs by its CD (cluster of differentiation) markers whiles MSCs was in passage two, such as CD45 as a negative marker and CD44 and CD73 as positive markers according to the previously reported (Piran et al. 2017).

\section{Cell culture}

After scaffolds sterilization using UV irradiation, adipose derived MSCs (AT-MSCs) at passage two were seeded on the scaffolds and tissue culture polystyrene (TCPS) for various experiments such as cell attachment assay $\left(10^{4}\right.$ per $\left.\mathrm{cm}^{2}\right)$, survival assay $\left(5 \times 10^{3}\right.$ per $\left.\mathrm{cm}^{2}\right)$ and study of the SMC differentiation potential $\left(2 \times 10^{4}\right.$ per $\left.\mathrm{cm}^{2}\right)$. Cell adhesion and survival assay were performed when cells grown under basal medium (DMEM/10\% FBS) and SMC differentiation was also done under basal medium supplemented with Ascorbic Acid $(30 \mathrm{mM}$, Merck, Germany) and TGF- $\beta 1$ ( 5 ng/mL, Peprotech, USA). Medium was exchanged every 2 days during the period of study.

\section{Survival assay}

To nontoxicity and biocompatibility evaluation of the fabricated scaffolds, AT-MSCs was cultured on the scaffolds and TCPS and on days 1, 5, 7 and 14 after cell seeding, cells were treated $4 \mathrm{~h}$ by $3-[4$, 5-dimethyl-thiazolyl-2]-2, 5-diphenol-tetrazoliumbromide solution (MTT, $5 \mathrm{mg} / \mathrm{mL}$ ). After this time, medium was removed and DMSO was added as a solvent of formed formazan crystal. After that, solutions optical density (OD) was read at $570 \mathrm{~nm}$ using a spectrophotometer.

\section{Real time RT-PCR}

To SMC related gene markers evaluation, on days 7 and 14 after differentiation induction, cells were detached from the scaffolds and TCPS and then their total RNA content was extracted using a total RNA Prep kit (Biofact, Daejeon, South Korea). After that RNA concentration was detected using the Nanodrop instrument (Nanodrop Technologies, Wilmington, $\mathrm{DE}$ ), and then certain volume of RNA was used for complementary DNA (cDNA) synthesizing using a cDNA synthesis kit (Biofact, Daejeon, South Korea). Finally, real time RT-PCR procedure was initiated by mixing of the certain volumes of injectable Water, SMC and internal control genes primers (Table 1) and 2X real-time PCR master mix (for SYBR Green, Biofact, Daejeon, South Korea) in a $0.2 \mathrm{~mL}$ micro tubes and placing on the ABI StepOnePlus thermal cycler (Applied Biosystems, Foster city, CA).

Immunocytochemistry analysis

To perform immunocytochemistry (ICC), at the end of study samples were fixed using $4 \%$ paraformaldehyde in PBS ( $\mathrm{pH} 7.4$ ) for $10 \mathrm{~min}$ at room temperature. After that, samples were washed three times with ice-cold PBS and then permeabilized by treating with PBS supplemented with $0.1-0.25 \%$ Triton $\mathrm{X}-100$ for $10 \mathrm{~min}$. Samples were washed again for three times ( 5 min every time) and then samples were blocked by $1 \%$ bovine serum albumin (BSA), $22.52 \mathrm{mg} / \mathrm{mL}$ glycine in PBST (PBS $+0.1 \%$ Tween 20) for $30 \mathrm{~min}$. Diluted primary antibody solution (Antismooth muscle Myosin heavy chain 11 antibody (ab125884) in 1\% BSA in PBST) was added to the

Table 1 The primers of the SMC related gene markers and an internal control gene that used in the real-time RT-PCR analysis at this study

\begin{tabular}{ll}
\hline Gene & Primer sequences $(5 \rightarrow 3)$ \\
\hline$\beta$-actin & GTCCTCTCCCAAGTCCACAC \\
aSMA & GGGAGACCAAAAGCCTTCAT \\
& GACGCACAACTGGCATC \\
SM22a & GVAGTVVAAAATTGAGA \\
& CTGTTGCTGCCCATTTGAAG \\
Caldesmon1 & AGACTACAAGGTATCATTGGAAC \\
& GACAGGTCAGCAATCAGATG \\
Collagen-I- $\alpha 1$ & GCCAAGGGTCTGACTGG \\
& CCCATCACACCAGCCTG \\
Collagen-III- $\alpha 1$ & CCAGGTGCTGATGGTGTC \\
& ACCTCTCTCACCAGGGCT \\
MHC & CAACCTGAGGGAGCGGTACTT \\
& GAGTAGATGGGCAGGTGTTTATAGG
\end{tabular}


samples and incubated in a humidified chamber at $4{ }^{\circ} \mathrm{C}$. Solution was removed from the samples and washed three times again, and finally incubated with a secondary antibody solution (Fluorescein isothiocyanate (FITC) conjugated goat anti-rabbit IgG (Invitrogen, Carlsbad, California) in 1\% BSA) for $1 \mathrm{~h}$ at room temperature. Samples were washed three times again and then DAPI (4',6-diamidino-2phenylindole; 1:1000) was added to the samples for nuclear staining and then samples were visualized using a florescent microscope.

Statistical analysis

All Experiments were done three times independently at this study and obtained data was reported as mean \pm standard deviation (SD). Real time RT-PCR data were analyzed by REST2009 software and other statistical analyses were performed using the SPSS software (version 19.0) by applying one-way ANOVA. Finally, probability values less than 0.05 were reported as significantly difference between groups (Table 2).

\section{Results}

Nanofibrous scaffold morphological characterization

Fabricated empty (Fig. 1a) and poly-P incorporated (Fig. 1b) scaffolds were studied morphologically using SEM and the results demonstrated that scaffolds were fibrous while fibers had diameters in nano-scale and bead free with interconnected pore. In addition poly-P incorporating didn't have any changed in scaffold's structure and fiber size and morphology. Scaffolds wettability and hydrophilicity were studied and the results showed that PLGA/PU contact angle was decreased significantly from $123^{\circ}$ to $82^{\circ}$ after incorporating with poly-P. After that, mechanical characteristics of the fabricated scaffolds was also studied using tensile assay and the results revealed that PLGA/PU tensile strength was decreased from $12.56 \pm 1.87$ to $10.35 \pm 0.66 \mathrm{MPa}$ after incorporating with poly-P, and PLGA/PU elongation at break was increased from $35.76 \pm 1.93$ to $43.21 \pm 2.38 \%$ after incorporating with poly-P.

Stem cell isolation and characterization

MSCs were isolated properly from abdominal fat tissue and AT-MSCs showed spindle shape with fibroblast like morphology at passage two (Fig. 2a), to confirm their mesodermal differentiation capacity, isolated cells cultured under adipogenic and osteogenic induction medium. Obtained results demonstrated that oil vesicles were observed absolutely 2 weeks after grown under adipogenic medium by oilred staining (Fig. 2b) and calcium deposition was also detected by alizarin-red staining 2 weeks after grown under osteogenic medium (Fig. 2c). In addition, morphology and shapes of the AT-MSCs while 2 weeks cultured under SMC induction medium was also changed properly (Fig. 2d) and become similar to the shape of the typical SMC. Flow cytometry results demonstrated that CD45 (Fig. 2e) was negative in 99.7\% of the isolated cells and CD73 (Fig. 2f) and CD44 (Fig. 2g) were also positive in 98.1 and $99.1 \%$ of the isolated cells, respectively.

Scaffolds biocompatibility evaluation

To biocompatibility evaluation of the fabricated PLGA/PU and PLGA/PU/poly-P nanofibrous scaffolds, protein adsorption (Fig. 3a) and cell attachment assay (Fig. 3b) were performed and results demonstrated that in both assays, the highest values was related to the PLGA/PU/poly-P scaffold. Increased protein adsorption and cell attachment was detected in

Table 2 Mechanical properties of the poly lactic-co-glycolic acid (PLGA)/polyurethane (PU) (B) and poly-phosphate (poly-P) incorporated PLGA/PU nanofibrous scaffold (PLGA/PU/poly-P) obtained from tensile assay

\begin{tabular}{llr}
\hline Group & Ultimate tensile strength (MPa) & Elongation at break (\%) \\
\hline PLGA/PU & $15.12 \pm 2.16$ & $32.13 \pm 0.57$ \\
PLGA/PU/polyP & $13.23 \pm 1.25$ & $39.45 \pm 1.78$ \\
\hline
\end{tabular}



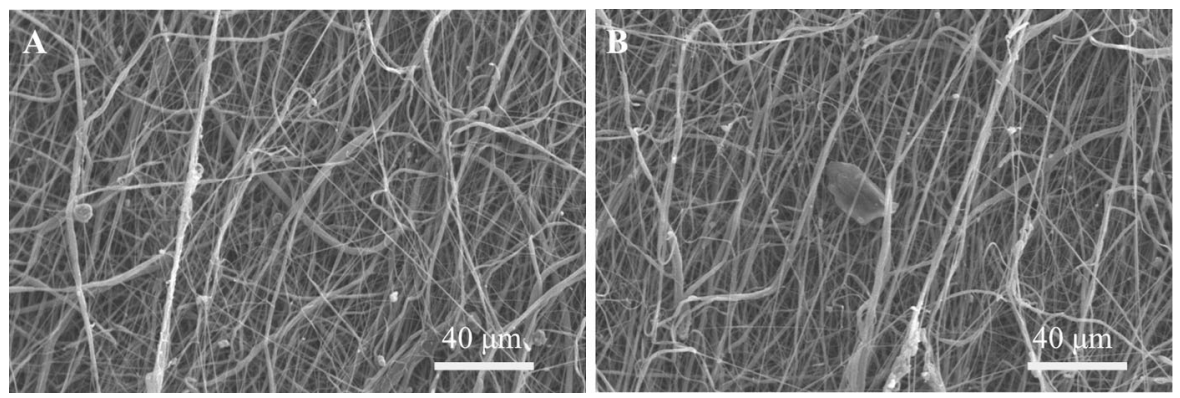

Fig. 1 SEM results: poly lactic-co-glycolic acid (PLGA) and polyurethane (PU) nanofibrous scaffold (a) and poly-phosphate (poly-P) incorporated PLGA/PU nanofibrous scaffold (PLGA/PU/poly-P) (b)
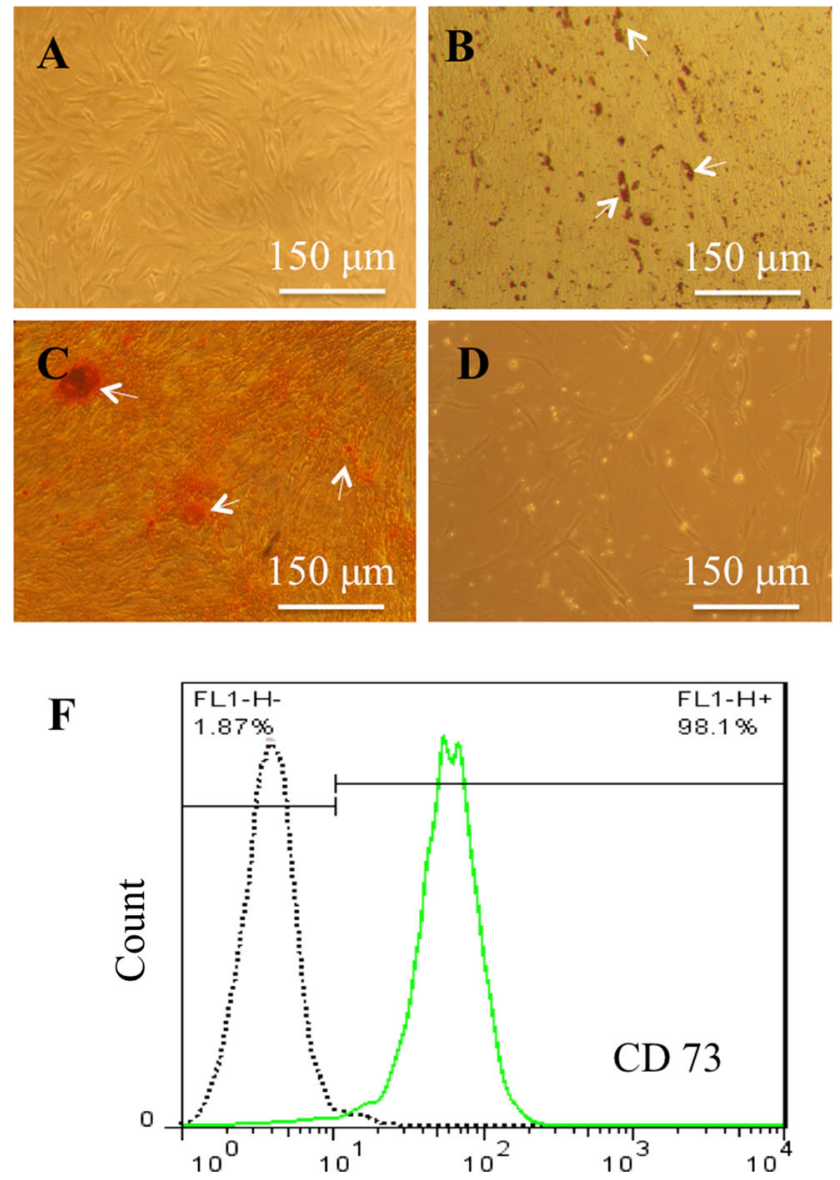

Fig. 2 Inverted light microscope results: human adipose derived mesenchymal stem cells (AT-MSCs) at passage three (a), human AT-MSCs after 2 weeks cultured under adipogenic induction medium while stained with oil-red (b), human ATMSCs after 2 weeks cultured under osteogenic induction

PLGA/PU while incorporated with poly-P that was significantly higher than PLGA/PU and TCPS groups. However these measures in PLGA/PU was also
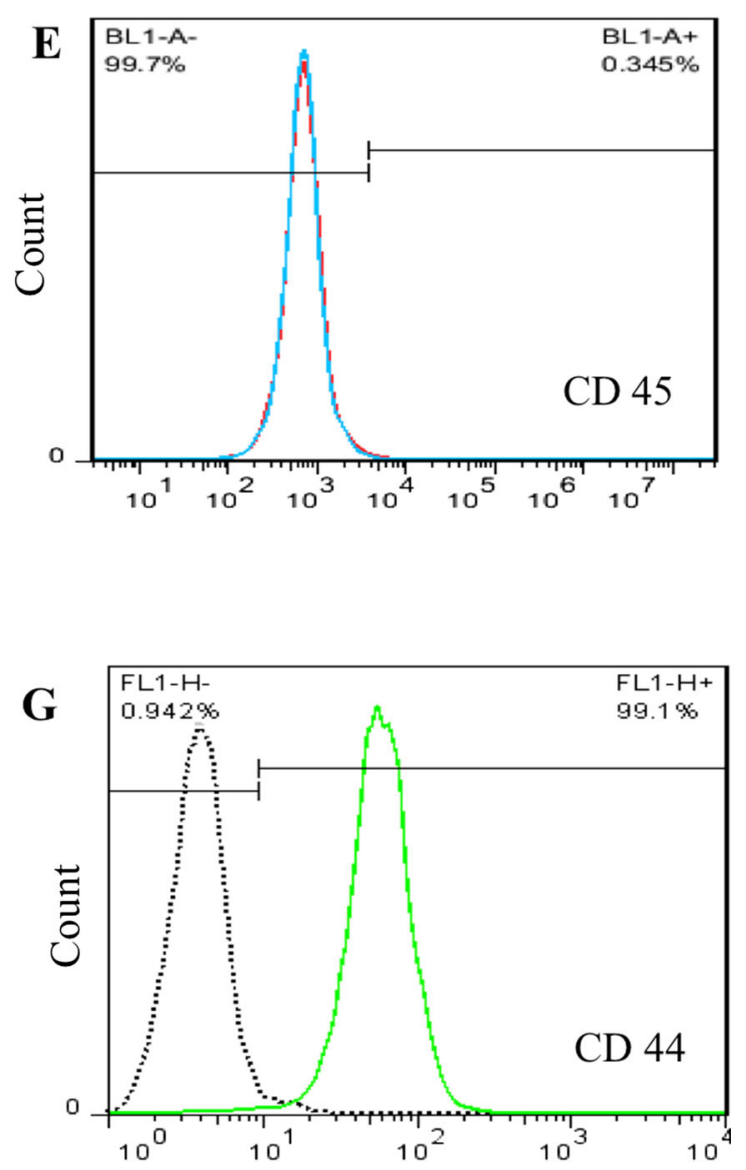

medium while stained with Alizarin-red (c) and human ATMSCs after 2 weeks grown under SMC induction medium (d). Flow cytometry analysis for isolated AT-MSCs: CD45 as a negative marker (e) and CD73 (f) and CD44 (g) as positive markers

significantly higher than measures detected in TCPS as a control. 


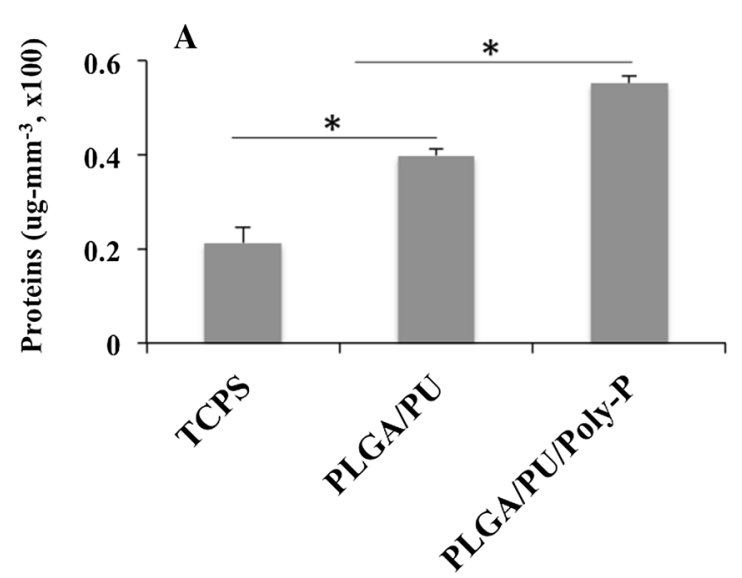

Fig. 3 Protein adsorption assay results for poly lactic-coglycolic acid (PLGA)/polyurethane (PU) and poly-phosphate (poly-P) incorporated PLGA/PU nanofibrous scaffold (PLGA/ $\mathrm{PU} /$ poly-P) and tissue culture polystyrene (TCPS) as a control

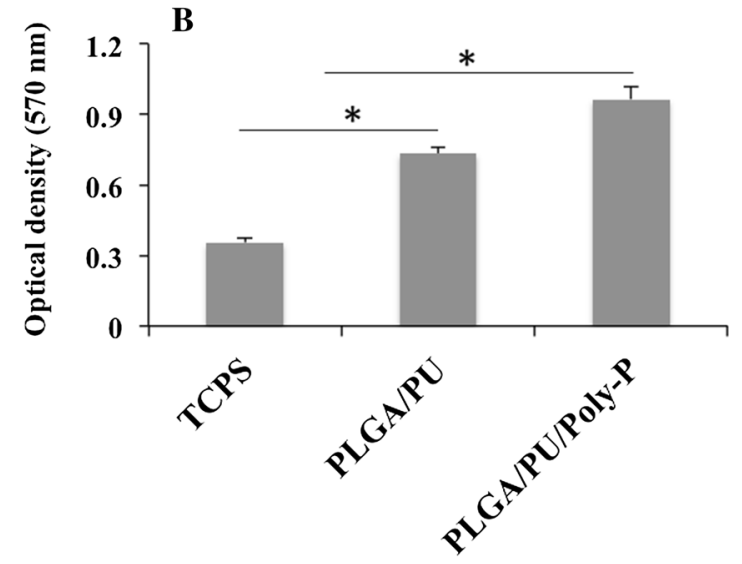

(a) and human adipose derived mesenchymal stem cells (ATMSCs) adhesion assay for PLGA/PU, PLGA/PU/poly-P and TCPS (b)
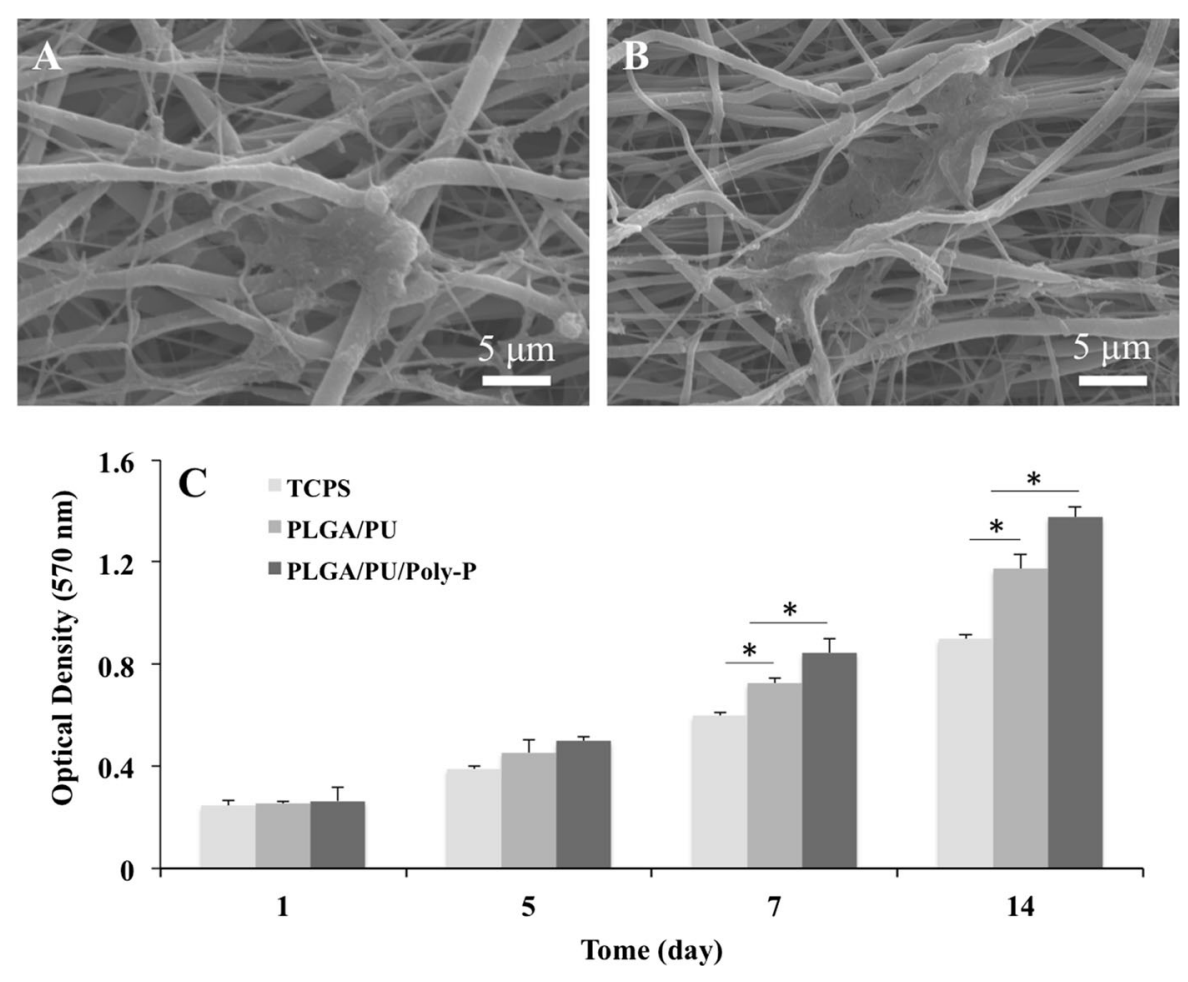

Fig. 4 SEM results: poly lactic-co-glycolic acid (PLGA)/ polyurethane (PU) (a) and poly-phosphate (poly-P) incorporated PLGA/PU (PLGA/PU/poly-P) (b) nanofibrous scaffold while human adipose derived mesenchymal stem cells (AT-

After that, cells adhesion, growth, proliferation and expansion on the surface of the fabricated scaffolds studied using SEM and results showed that AT-MSCs
MSCs) cultured on them. Viability assay results for the human AT-MSCs while cultured on PLGA/PU, PLGA/PU/poly-P and TCPS as a control $(\mathbf{c})$. The significant differences $(P<0.05)$ between groups are indicated with star sign

properly attached and proliferated on the surface of the PLGA/PU (Fig. 4a) and PLGA/PU/poly-P (Fig. 4b) nanofibrous scaffolds. AT-MSCs proliferation and 
survival rate when grown on the scaffolds was also studied by MTT assay (Fig. 4c) during 2 weeks and obtained results revealed that there was not a significant difference between AT-MSC survival rate on days 1 and 5, although on day 5 it was higher in PLGA/ PU/poly-P scaffold than PLGA/PU scaffold and so an compared to the TCPS. But, the highest survival rate of AT-MSC on days 7 and 14 was detected while grown on PLGA/PU/polyP scaffold that was significant compared to the other groups. On these 2 days, the survival rate of AT-MSC while grown on PLGA/ PU scaffold was also significantly higher than those cells grown on TCPS group.

SMC related gene expression

To evaluate SMC supportive potential of fabricated nanofibrous scaffolds, SMC differentiation potential of the AT-MSC was studied while grown in the different groups using SMC related genes expression measurement (Fig. 5). The results demonstrated that SM22-a, ASMA and Calponin1 showed a similar expression pattern during 2 weeks, where on both 7 and 14 days after differentiation induction, the highest expression level of these genes was detected in ATMSC cultured on PLGA/PU/poly-P scaffold that was significantly higher than others. In addition, these genes in AT-MSC cultured on PLGA/PU scaffold were also expressed significantly higher than those cells cultured in TCPS group. The expression level of MHC was increased significantly on day 7 in AT-MSC cultured on PLGA/PU/poly-P scaffold, while in other two groups the difference wasn't significant. Although, on day 14, the highest significant difference in MHC expression was detected in AT-MSC cultured on PLGA/PU/poly-P scaffold, and this gene in ATMSC cultured on PLGA/PU scaffold was also expressed significantly higher than TCPS group. In following, Col-I and Col-III expression levels were also evaluated and the results demonstrated that on both days, the highest expression level of Col-I was observed in AT-MSC cultured on PLGA/PU/poly-P scaffold, where on day 7 expression level of this gene in AT-MSC cultured on PLGA/PU and TCPS groups wasn't significantly different, but on day 14 this gene in AT-MSC cultured on PLGA/PU scaffold was expressed significantly higher than TCPS group. Col-III expression level in different groups was not changed significantly on both days, only on day 14 this gene in AT-MSC cultured on PLGA/PU/poly-P scaffold was expressed significantly higher than other groups.

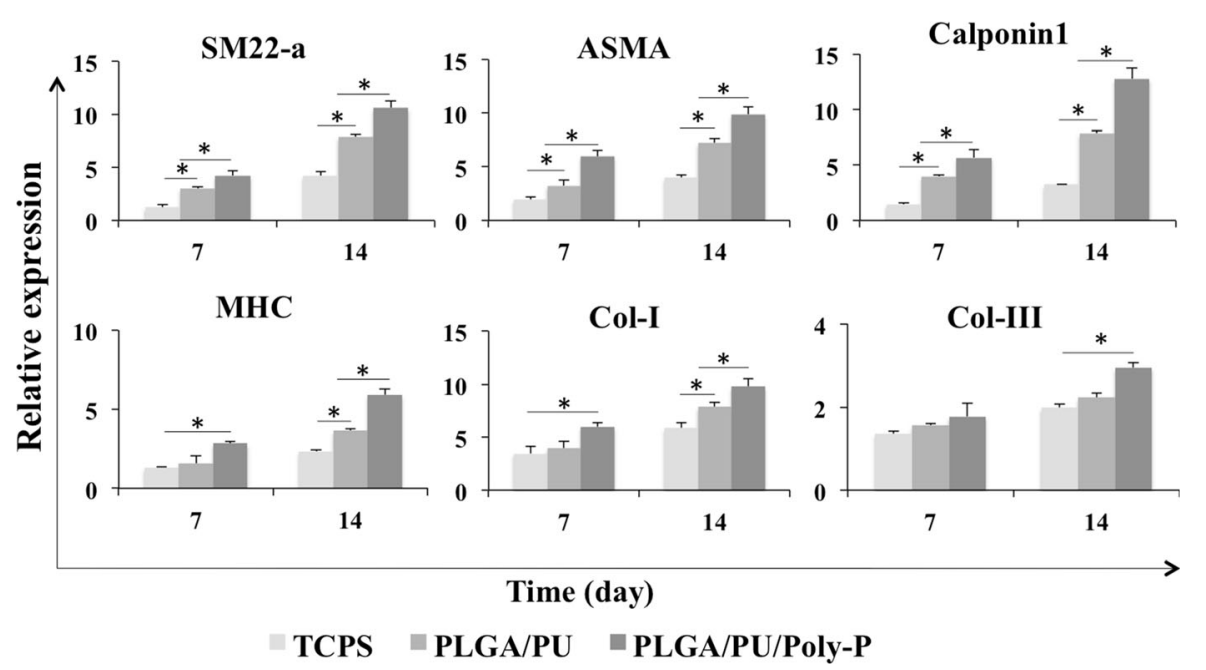

Fig. 5 Real-time RT-PCR results: relative expression of Smooth muscle 22 alpha (SM-22a), alpha-smooth muscle actin (ASMA), calponi1, myosin heavy chain (MHC), collagen type I (Col-I) and collagen type III (Col-III) in human adipose derived mesenchymal stem cells (AT-MSCs) cultured on poly lactic-co- glycolic acid (PLGA)/polyurethane (PU) and poly-phosphate (poly-P) incorporated PLGA/PU nanofibrous scaffold (PLGA/ $\mathrm{PU} /$ poly-P) and tissue culture polystyrene (TCPS) as a control. The significant differences $(P<0.05)$ between groups are indicated with star sign 
SMC related protein expression

For more detailed evaluation of the SMC-differentiation efficiency of the AT-MSC while cultured on the different substrates, one of the most important SMC related protein including smooth muscle MHC was studied using ICC at the end of study. The results demonstrated that this protein was expressed in ATMSC cultured on PLGA/PU/poly-P scaffold (Fig. 6c) higher than AT-MSC cultured on PLGA/PU scaffold (Fig. 6b) and TCPS (Fig. 6a). It seems that this protein in AT-MSC cultured on TCPS was expressed significantly lower than other two groups. SMC-differentiated AT-MSC cultured on TCPS (Fig. 6d) and PLGA/ PU (Fig. 6e) and PLGA/PU/poly-P (Fig. 6f) scaffolds was also stained by DAPI.

\section{Discussion}

Tissue engineering structures designed to repair damaged tissue can include three parts, a cell, a scaffold and a biologically active factor that can accelerate the process of cells differentiation into the target tissue (Hosseinkhani et al. 2014). Depending on the target tissue, sections can be changed. Recently, several studies have confirmed the possibility of bladder smooth muscle remodeling using engineered constructs made from native and synthetic biomaterials cultured with cells in vitro (Atala 2011; Corona et al. 2013; Drewa et al. 2006). In this project, a novel system of electrospun bioactive scaffold is presented for the bladder tissue engineering with the aim of supporting in vitro SMC differentiation. It was demonstrated that RhoA and AKT pathways play a critical role in SMC differentiation process (Mack 2011), on the other hand Hassanian et al. were also reported that poly-P has a great role in activation of the mTOR signaling where the interaction of poly-P with RAGE and P2Y1 activates mTORC1 pathway by PI3K/AKT activation (Hassanian et al. 2016). In this because, we decided to incorporate poly-P as a bioactive molecules in the biodegradable PLGA/PU nanofibrous scaffold, since poly-P can released continuously from the nanofibers and can trigger mTOR pathway in the cells while cultured on the nanofibrous scaffold. This release along with SMC induction medium can enhance the efficacy of the SMC differentiation. The results achieved at this study demonstrated that polyP incorporating in PLGA/PU nanofibers didn't have any significant change in the scaffold's structural and morphological characteristics, while PLGA/PU scaffold's elongation at break, protein adsorption, cell adhesion, wettability and
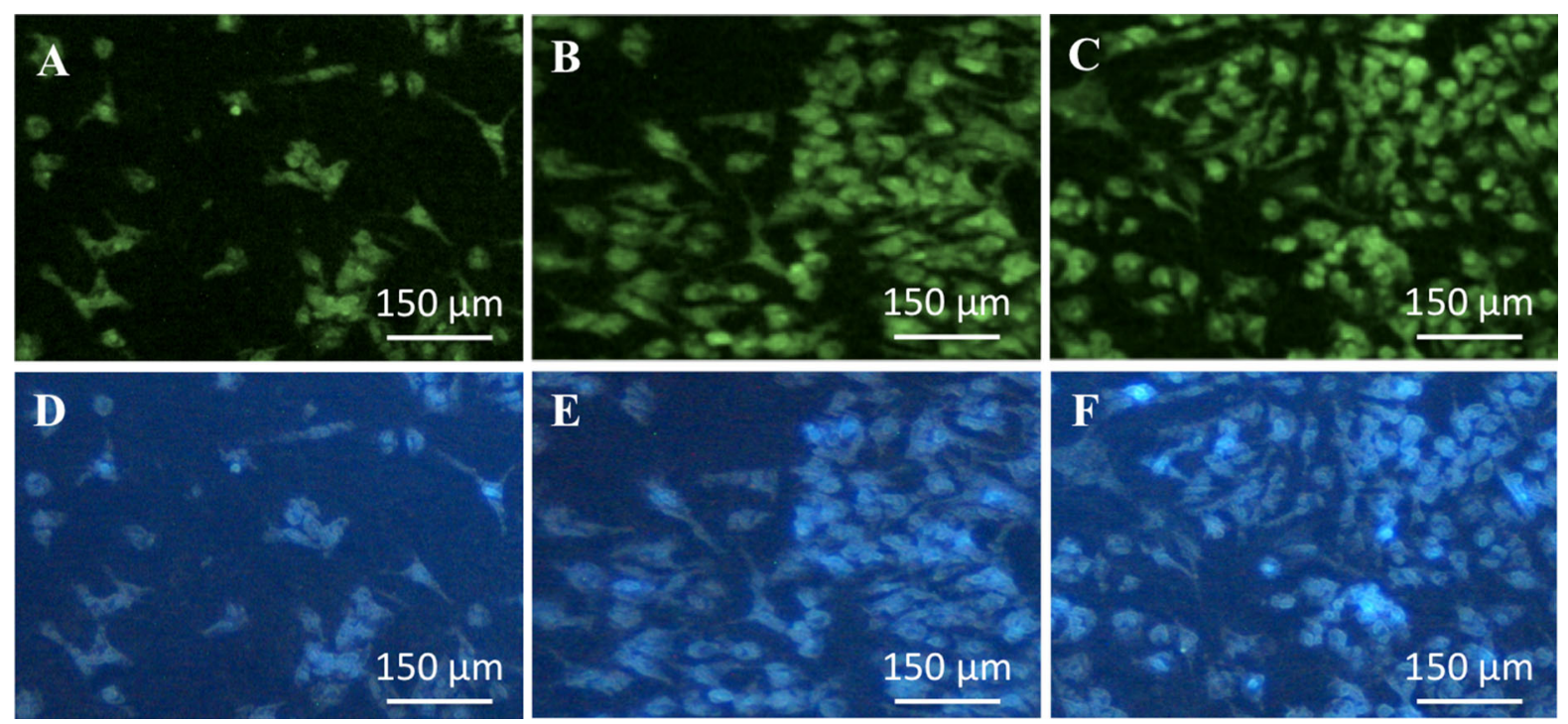

Fig. 6 Immunocytochemistry assay results: bladder myosin heavy chain (MHC) protein in human adipose derived mesenchymal stem cells (AT-MSCs) cultured on tissue culture polystyrene (TCPS) (a), poly lactic-co-glycolic acid (PLGA)/ polyurethane (PU) (b) and poly-phosphate (poly-P) incorporated PLGA/PU nanofibrous scaffold (PLGA/PU/poly-P) (c). DAPI staining for determination of cell's nucleus cultured on TCPS (d), PLGA/PU (e) and PLGA/PU/poly-P (f) groups 
biocompatibility were increased after incorporating with poly-P. In agree with our results, it was demonstrated previously the presence of the poly-P cases to increase in cell proliferation and survival (Hosseini et al. 2019; Rao et al. 2009). In following, AT-MSCs were cultured on the PLGA/PU/poly-P and PLGA/PU nanofibrous scaffolds and TCPS as a control under SMC differentiation medium to investigate SMC supportive capacity of the fabricated scaffolds. Obtained results at the gene and protein levels demonstrated that fabricated PLGA/PU has a great SMC supportive capacity when AT-MSCs cultured on it compared to the TCPS. In addition, this supportive potential of PLGA/PU from SMC differentiation of AT-MSCs was significantly increased when these fibers filled by poly-P that can be related to the polyP activity in triggering mTOR and AKT signal pathways that are involved in SMC modulation (Martin et al. 2004; Mourani et al. 2004). In agreement with our results, mTOR signaling has been shown to be involved in regulating cell growth and proliferation in response to cellular environments, including adequate nutrients, energy, and oxygen (Martin and Blenis 2002; Sarbassov et al. 2005). In addition, Rzucidlo et al. (2007) demonstrated that AKT signal pathway play a critical role in vascular SMC differentiation.

\section{Conclusion}

Our results demonstrated that fabricated PLGA/PU nanofibrous scaffold has a great potential to support SMC differentiation of the human MSCs. Since there is no definitive cure for bladder disease due to bladder wall functional disorders, the use of this scaffold and poly-P may be helpful in assisting such patients when used as a bio-implant in the treatment of bladder smooth muscle damages. In addition, while poly-P incorporated in PLGA/PU nanofibers, this supportive property will be increased according to the results presented at this study. It can be concluded that, based on the poly-P role in mTOR/AKT signal pathways and the role of these pathways in SMC differentiation, it could be related to the synergistic effect of these two parts of our fabricated scaffolds including scaffold topography and incorporated poly-P as a biological active molecule.
Acknowledgements Shahid Beheshti University of Medical Sciences was financially supported from this study (Grant No. 18712).

\section{Compliance with ethical standards}

Conflict of interest The authors declare that there is no conflict of interests.

\section{References}

Alghoraibi I, Alomari S (2018) Different methods for nanofiber design and fabrication. In: Handbook of nanofibers, pp $1-46$

Amani H, Arzaghi H, Bayandori M, Dezfuli AS, Pazoki-Toroudi H, Shafiee A, Moradi L (2019) Controlling cell behavior through the design of biomaterial surfaces: a focus on surface modification techniques. Adv Mater Interfaces 6:1900572

Ardeshirylajimi A et al (2016) Fat harvesting site is an important determinant of proliferation and pluripotency of adiposederived stem cells. Biologicals 44:12-18

Ardeshirylajimi A, Golchin A, Khojasteh A, Bandehpour M (2018) Increased osteogenic differentiation potential of MSCs cultured on nanofibrous structure through activation of Wnt/ $\beta$-catenin signalling by inorganic polyphosphate. Artif Cells Nanomed Biotechnol 46:S943-S949

Ardeshirylajimi A, Golchin A, Vargas J, Tayebi L (2020) Application of stem cell encapsulated hydrogel in dentistry. In: Applications of biomedical engineering in dentistry. Springer, pp 289-300

Atala A (2011) Tissue engineering of human bladder. Br Med Bull 97:81-104

Corona BT, Ward CL, Baker HB, Walters TJ, Christ GJ (2013) Implantation of in vitro tissue engineered muscle repair constructs and bladder acellular matrices partially restore in vivo skeletal muscle function in a rat model of volumetric muscle loss injury. Tissue Eng Part A 20:705-715

Dedkova EN, Blatter LA (2014) Role of $\beta$-hydroxybutyrate, its polymer poly- $\beta$-hydroxybutyrate and inorganic polyphosphate in mammalian health and disease. Front Physiol 5:260

Drewa T, Sir J, Czajkowski R, Wozniak A (2006) Scaffold seeded with cells is essential in urothelium regeneration and tissue remodeling in vivo after bladder augmentation using in vitro engineered graft. In: Transplantation proceedings. Elsevier, pp 133-135

Franco I (2012) Functional bladder problems in children: pathophysiology, diagnosis, and treatment. Pediatr Clin 59:783-817

Ghiaee A, Pournaqi F, Vakilian S, Mohammadi-Sangcheshmeh A, Ardeshirylajimi A (2017) Adapted dexamethasone delivery polyethylene oxide and poly ( $\varepsilon$-caprolactone) construct promote mesenchymal stem cells chondrogenesis. Artif Cells Nanomed Biotechnol 45:1640-1648

Ghorbani FM, Kaffashi B, Shokrollahi P, Seyedjafari E, Ardeshirylajimi A (2015) PCL/chitosan/Zn-doped nHA electrospun nanocomposite scaffold promotes adipose 
derived stem cells adhesion and proliferation. Carbohydr Polym 118:133-142

Golchin A, Farahany TZ, Khojasteh A, Soleimanifar F, Ardeshirylajimi A (2019) The clinical trials of mesenchymal stem cell therapy in skin diseases: an update and concise review. Curr Stem Cell Res Ther 14:22-33

Griffith LG, Naughton G (2002) Tissue engineering-current challenges and expanding opportunities. Science 295:1009-1014

Hassanian SM, Ardeshirylajimi A, Dinarvand P, Rezaie AR (2016) Inorganic polyphosphate promotes cyclin D1 synthesis through activation of mTOR/Wnt/ $\beta$-catenin signaling in endothelial cells. J Thromb Haemost 14:2261-2273

Hassanian SM, Avan A, Ardeshirylajimi A (2017) Inorganic polyphosphate: a key modulator of inflammation. J Thromb Haemost 15:213-218

Hoag N, Gani J (2015) Underactive bladder: clinical features, urodynamic parameters, and treatment. Int Neurourol J 19:185

Hosseini FS, Soleimanifar F, Khojasteh A, Ardeshirylajimi A (2019) Promoting osteogenic differentiation of human-induced pluripotent stem cells by releasing $\mathrm{Wnt} / \beta$-catenin signaling activator from the nanofibers. $\mathrm{J}$ Cell Biochem 120:6339-6346

Hosseinkhani M, Mehrabani D, Karimfar MH, Bakhtiyari S, Manafi A, Shirazi R (2014) Tissue engineered scaffolds in regenerative medicine. World J Plast Surg 3:3

Khalf A, Madihally SV (2017) Recent advances in multiaxial electrospinning for drug delivery. Eur J Pharm Biopharm 112:1-17

Lanza R, Langer R, Vacanti JP (2011) Principles of tissue engineering. Academic Press, Cambridge

Laschke MW et al (2009) In vivo biocompatibility and vascularization of biodegradable porous polyurethane scaffolds for tissue engineering. Acta Biomater 5:1991-2001

Lorenz B, Kurz L, Müller WEG (2012) Inorganic polyphosphate in eukaryotes: enzymes, metabolism and function. Inorg Polyphosphates Biochem Biol Biotechnol 23:45

Mack CP (2011) Signaling mechanisms that regulate smooth muscle cell differentiation. Arterioscler Thromb Vasc Biol 31:1495-1505

Mao F, Tu Q, Wang L, Chu F, Li X, Li HS, Xu W (2017) Mesenchymal stem cells and their therapeutic applications in inflammatory bowel disease. Oncotarget 8:38008

Martin KA, Blenis J (2002) Coordinate regulation of translation by the PI 3-kinase and mTOR pathways. Adv Cancer Res 86:2-41

Martin KA, Rzucidlo EM, Merenick BL, Fingar DC, Brown DJ, Wagner RJ, Powell RJ (2004) The mTOR/p70 S6K1 pathway regulates vascular smooth muscle cell differentiation. Am J Physiol Cell Physiol 286:C507-C517
Mourani PM, Garl PJ, Wenzlau JM, Carpenter TC, Stenmark KR, Weiser-Evans MCM (2004) Unique, highly proliferative growth phenotype expressed by embryonic and neointimal smooth muscle cells is driven by constitutive Akt, mTOR, and p70S6K signaling and is actively repressed by PTEN. Circulation 109:1299-1306

Park H, Lee KY, Lee SJ, Park KE, Park WH (2007) Plasmatreated poly (lactic-co-glycolic acid) nanofibers for tissue engineering. Macromol Res 15:238-243

Piran M, Enderami SE, Piran M, Sedeh HS, Seyedjafari E, Ardeshirylajimi A (2017) Insulin producing cells generation by overexpression of $\mathrm{miR}-375$ in adipose-derived mesenchymal stem cells from diabetic patients. Biologicals 46:23-28

Rao NN, Gómez-García MR, Kornberg A (2009) Inorganic polyphosphate: essential for growth and survival. Ann Rev Biochem 78:605-647

Rzucidlo EM, Martin KA, Powell RJ (2007) Regulation of vascular smooth muscle cell differentiation. J Vasc Surg 45:A25-A32

Sarbassov DD, Ali SM, Sabatini DM (2005) Growing roles for the mTOR pathway. Curr Opin Cell Biol 17:596-603

Schmidt RA (2008) Use of neurotoxin therapy for treatment of urologic and related disorders related to lowering elevated bladder pressure. Google Patents

Schröder HC, Müller WEG (2012) Inorganic polyphosphates: biochemistry, biology, biotechnology, vol 23. Springer, Berlin

Sill TJ, von Recum HA (2008) Electrospinning: applications in drug delivery and tissue engineering. Biomaterials 29:1989-2006

Solesio ME, Demirkhanyan L, Zakharian E, Pavlov EV (2016) Contribution of inorganic polyphosphate towards regulation of mitochondrial free calcium. Biochimi Biophys Acta (BBA) Gen Subj 1860:1317-1325

Subbiah T, Bhat GS, Tock RW, Parameswaran S, Ramkumar SS (2005) Electrospinning of nanofibers. J Appl Polym Sci 96:557-569

Wang H-S, Fu G-D, Li X-S (2009) Functional polymeric nanofibers from electrospinning. Recent Patents Nanotechnol 3:21-31

Zhu G-Q, Wang F-G, Tan H-S, Gao Q-C, Liu Y-Y (2014) Properties of poly (lactic acid-co-glycolic acid) film modified by blending with polyurethane. Chem Pap 68:246-252

Publisher's Note Springer Nature remains neutral with regard to jurisdictional claims in published maps and institutional affiliations. 